\title{
La voix suspecte : des crocodiles et des hommes
}

\section{Margaret Jones-Davies}

\section{Q OpenEdition \\ Journals}

\section{Édition électronique}

URL : http://journals.openedition.org/shakespeare/375

DOI : 10.4000/shakespeare.375

ISSN : 2271-6424

\section{Éditeur}

Société Française Shakespeare

\section{Édition imprimée}

Date de publication : 1 novembre 1999

Pagination : 131-158

ISBN : 2-84269-331-0

\section{Référence électronique}

Margaret Jones-Davies, "La voix suspecte : des crocodiles et des hommes », Actes des congrès de la Société française Shakespeare [En ligne], 17| 1999, mis en ligne le 01 novembre 2007, consulté le 22 avril 2019. URL : http://journals.openedition.org/shakespeare/375 ; DOI : 10.4000/shakespeare.375 


\section{S H A K E S P E A R E \\ \& $\quad$ L $A \quad$ V O I X}

Société Française Shakespeare

Actes du Congrès de 1999

米米

Textes réunis et présentés par

Patricia DORVAL

publiés sous la direction de

Jean-Marie MAGUIN 


\section{LA VOIX SUSPECTE: DES CROCODILES ET DES HOMMES}

Lorsque Dieu dans le Timée créa l'homme, il veilla à ce que les parties du corps qui renferment de l'intelligence ne soient pas rendues insensibles par des «chairs abondantes, éparses et fortement tassées» (74e). Exception est faite pour cette «masse de chair» (75a) qu'est la langue. C'est que «l'appareil de la bouche» fait de «dents, d'une langue et de lèvres» a été disposé «comme il l'est actuellement en vue du nécessaire et en vue du bien... car tout ce qui entre pour fournir sa nourriture au corps est nécessaire et le courant de paroles qui sort de nos lèvres pour le service de l'intelligence est le meilleur de tous les courants» (75e). C'est un peu à contre cœur que Platon fait l'éloge de cette masse de chair qu'est la langue, organe à double fonction, double voie, organe équivoque où passe la nourriture terrestre et céleste, organe d'une frontière entre le corps et l'âme : le danger d'une contamination est grand.

Dans La République, l'ouïe et la voix sont hiérarchiquement inférieurs aux yeux (livre VI.507c-d) car l'ouvrier des sens s'est mis moins en dépense pour fabriquer l'ouie et la voix qui n'ont pas besoin d'une autre chose d'espèce différente pour fonctionner alors que les yeux ont besoin de la lumière. Mais dans le Timée, Platon décrit pourtant l'importance de l'air dans la production du son, défini comme un coup donné par l'air à travers les oreilles au cerveau et au sang arrivant jusqu'à l'âme (67b). 
Platon justifie ainsi ce qu'il ne peut sans doute pas s'empêcher dans son for intérieur de considérer comme des défauts de fabrication. Si les organes de la parole ne sont pas explicitement suspects, l'usage de la parole l'est beaucoup plus. La voix est suspecte parce qu'elle peut trop facilement franchir les limites de ce qui définit l'homme à cause se son pouvoir d'imitation. Aristote souligne ce pouvoir dans sa Rhétorique : " la voix est le plus propre à l'imitation» (livre III.20.1404a). La voix est suspecte parce que non seulement elle peut imiter «le bruissement des fleuves, le ressac de la mer, les coups de tonnerre et tous les bruits de ce genre» mais aussi «le hennissement des chevaux, le mugissement des taureaux» (Rép., livre III.396b). Par la voix, l'homme ne peut-il pas perdre sa noblesse, chuter dans l'échelle des êtres, cesser d'être le parangon des animaux ? Le mauvais orateur sera porté à tout imiter... «coups de tonnerre, bruits du vent, orages, bruits d'essieux et de poulies, sons de trompettes, de flûtes, de flûtes de pan et de tous les instruments et encore cris de chiens, de moutons et d'oiseaux; ainsi sa diction tout entière se fera par imitation à grand renfort de voix et de gestes» (Rép., livre III.397a-b).

La voix est le médium par excellence de l'imitation. Elle est suspecte parce qu'elle peut imiter les bruits inhumains de la folie. C'est pourquoi il est formellement interdit «aussi bien de délirer que de se rendre semblables à ceux qui délirent (livre III.396.b) D'ailleurs les rôles d'acteurs seront réservés aux esclaves et aux étrangers (Lois, VII.816e) (voir note 9). Lorsque Shakespeare met en scène les grands imitateurs de la folie - Hamlet, Edgar - c'est le discours de Platon sur le danger que représente la voix que l'on entend.

La voix peut faire basculer l'assise de la raison, elle est ce qui nous rapproche le plus de l'animal par sa capacité à l'imiter. Dans le De Anima, Aristote souligne la différence essentielle entre «le son émis par l'animal» qui «n'est pas voix [...] car la voix est assurément un son pourvu de signification, et elle n'est pas uniquement le bruit de l'air respiré, comme la toux...» (420b.30). Dans le contexte platonicien, l'imitation semble faire perdre à la voix sa spécificité : elle est contamination. Son origine, dès lors, peut se trouver dans des cavités du corps moins proches du cerveau que la bouche. Selon Reginald Scot, dans The Discoverie of Witchcraft (livre VII) la voix de Satan provient du ventre. Les Pythonesses étaient ventriloques. Le mot hébreu $o b$ traduit par pytho signifie bouteille, car les Pythonesses ont une voix creuse comme une bouteille. La voix s'animalise, les sorcières parlent par leurs familiers. «Paddock calls» (Macbeth, I.1.9) nous dit la deuxième 
sorcière de Macbeth. On l'entend hululer sur toute la gamme des modulations qui passent de «hollow» à «howl» - cet adjectif et ce verbe reliés par un effet de paronomase ponctuent souvent la description des métamorphoses vocales de Shakespeare. Comme les voix schizophrènes décrites par Artaud, et qui portent un sens qui "vient d'affre»", la voix aux origines instables fait peur et les frontières corporelles de la voix sont si indéterminées qu'elles pourraient bien remettre en cause les formes essentielles définies comme immuables par la théologie. Dans son chapitre de Mille Plateaux «Devenir-intense, devenir-animal, devenir-imperceptible», Deleuze insiste sur l'aspect territorialisé de la théologie : «la théologie est très stricte sur le point suivant : il n'y a pas de loupsgarous, l'homme ne peut pas devenir animal» ${ }^{2}$. Et pourtant il partage avec l'animal ces organes producteurs de voix suspectes. Consacrés par une symbolisation adéquate, ces organes bestiaux peuvent servir la gloire divine. Dans une marqueterie de Lorenzo Lotto, par exemple, la mâchoire d'âne des Philistins devient le symbole dans la lecture éthique de l'épisode biblique de Samson de l'éloquence des prédicateurs.

Comment ne pas déifier alors ces animaux qui n'ont pas de voix : Plutarque rapporte dans Isis et Osiris que le crocodile est sacré chez les Égyptiens car il n'a pas de langue et pour cette raison symbolise le Verbe divin qui n'a pas de voix ${ }^{3}$. Le crocodile a bel et bien une langue, mais selon l'Encyclopédie britannique, elle est en grande partie reliée au plancher de la bouche et n'est pas déliée. Shakespeare s'inspire de la tradition platonicienne et hermétique qui se méfie de la voix. On verra cependant les limites de cette suspicion car il suivra aussi cet aspect de la tradition qui valorise la voix de par sa proximité avec le Verbe.

\section{Hamlet sous le signe du crocodile ?}

La voix de Hamlet en imitant la folie transgresse la mise en garde de Platon. De cette transgression découle l'effacement de toutes les frontières de l'identité. Le royaume de Danemark devient celui des métamorphoses et ses remparts confinent aux frontières du royaume des morts. Ce n'est pas un hasard si c'est dans cette pièce que le problème de la définition de l'homme se pose de la manière la plus aiguë. L'identité de Hamlet vacille devant l'ombre paternelle qu'il voit tantôt sublimée dans la noblesse, tantôt rabaissée dans le grotesque. Même si la tradition théâtrale et cinématographique a le plus souvent donné à la voix du fantôme des tonalités quasi surnaturelles, amples, empreintes de dignité, elle n'est que la voix 
de la taupe aveugle qui vient des profondeurs du «cellarage» (Hamlet, 1.5.159). Rien n'est plus naturel, plus corporel, plus animal que cette voix de l'au-delà, soi disant surnaturelle. Elle n'a rien à voir avec «the ear deafening voice of th' oracle» (The Winter's Tale, III.1.9) qui clame une vérité indéniable. N'est-ce pas après tout le fracas de la mer en bas de la falaise qu'elle mime? Platon prévenait contre l'imitation du bruit de la mer parce que ce dernier peut comme le dit Horatio nous faire basculer dans la folie (Q2. I.4.54 et suivantes). De même dans King Lear, Tom o' Bedlam se rapproche du bord de la falaise qui surplombe la mer pour mieux mimer la folie, si semblable à l'inhumain de la mer (King Lear, IV.6). En imitant la folie et son langage, la voix de Hamlet ne transgresse pas seulement l'ordre platonicien, il transgresse aussi la règle du silence inscrite dans le Poimandrès le premier livre du Corpus Hermeticum, très proche de l'évangile de saint Jean qui fait du Verbe le principe de toute chose et qui parle de «cet inexpressible, cet ineffable que le silence seul peut nommer» ${ }^{4}$. Dans le Poimandrès la voix humaine doit se taire devant la voix de la lumière qui sort des ténèbres comme un «cri inarticulé» 5 .

La voix du fantôme n'a rien de commun avec cette voix de la lumière qui se confond avec l'Intelligible platonicien, la Forme ou le Père. Le fantôme n'est qu'une caricature de cette Forme : il n'est que «a questionable shape» (Hamlet, I.4.43). En conséquence, son incarnation dans la matière que symbolise le passage à la voix, n'aboutit pas à la génération du cosmos ordonné selon le Logos, mais à l'assombrissement dans la folie feinte et réelle. Toute la pièce renverse la parabole de la création du Poimandrès. Dans Hamlet, la voix se meurt non pas dans le silence sacré du crocodile, mais dans le silence du meurtre : «For Murder hath no tongue...» même si la suite de la citation nous dit : «but it will speak with most miraculous organ» (Hamlet, II.2.582-3). Un silence lourd de secrets refoulés plane sur Elsinore. L'emblème de la pièce est le crâne de Yorick, «that had a tongue in it» (Hamlet, V.1.72). Le fou n'a plus de voix pour libérer sous forme d'énigmes et de paradoxes les vérités cachées. Dans ce contexte les mots que l'on entend ne génèrent que du silence. «I have words to speak in thine ear will make thee dumb» (Hamlet, IV.7.23). La parole du fantôme n'est pas génératrice d'une vérité harmonieuse dont on verrait les fruits dans une manifestation du réel. Ce n'est que la voix de la tentation qu'il convient de ne pas écouter comme il conviendra de ne pas écouter les voix des sorcières dans Macbeth. La soi-disant lâcheté de Hamlet qui tarde à donner corps aux paroles du fantôme n'est que 
prudence, la prudence qui anoblit l'acte et qu'illustre dans l'Iliade le dialogue de Diomède et Glaucos qui aboutit à l'interruption de leur combat (Iliade, VI.119.236). Dans Hamlet on voit que le corps désormais ne répond plus au Logos et commande donc à l'esprit de souffrir avec lui (King Lear, II.4.108-9). D'où ces nombreux dumb shows emblématisés par le spectacle donné au roi (Hamlet, III.2). L'actio n'y est plus que gesta sans voix qui miment la folie, par exemple, la scène où Hamlet rencontre Ophélie et sans un mot devient aussi terrifiant que le fantôme qu'il semble mimer (Hamlet, II.1.88-101), ou encore cet autre dumb show (Hamlet, IV.5) où voix et gestes seront irrémédiablement divorcés dans la folie d'Ophélie (Hamlet, IV.5.4-13).

L'actio qui normalement se réfère à la voix aussi bien qu'aux gestes avait une éthique fondée sur l'analogie des mouvements du corps avec ceux de l'âme - mais comme le dit Zumthor dans La Lettre et la Voix, l'Église veillait à «modérer et à contenir, au nom de la vertu l'activité gestuelle» ${ }^{6}$. Il y eut même au XIVème siècle une décrétale de Jean XXII, Docta sanctorum patrum, qui condamnait les gestes qui doublent en les mimant les paroles chantées ${ }^{7}$. Lorsqu'il y a divorce entre la gestuelle et la parole, le lien ne se fait plus avec le Logos. La suivante de Lady Macbeth rapporte au docteur une description de ses gestes mais se refuse à rapporter les mots de la somnambule. La gestuelle séparée des mots exprime le désordre et le chaos du corps comme dans le Tiers Livre de Rabelais qui interprète en termes sexuels tous les gestes du «conseil des muetz» (chap. XIX).

Mais dans Titus Andronicus, par exemple, on oppose deux types de dumb shows, ceux qui proviennent d'un silence désespéré :

Titus. Shall we bite our tongues in dumb shows Pass the remainder of our hateful days ?

(Titus Andronicus, III.1.131)

et les dumb shows où cette actio rendue muette est une vraie partie de la rhétorique qui substitue son éloquence par nécessité à la voix manquante et atteignant une extraordinaire perfection de l'expression se trouve donc légitimée :

Titus. Speechless complainer, I will learn thy thought;

In thy dumb action will I be as perfect

As begging hermits in their holy prayers.

Thou shalt not sigh, nor hold thy stumps to heaven 
Nor wink, nor nod, nor kneel, nor make a sign But I of these will wrest an alphabet

And by still practice learn to know thy meaning

( Titus Andronicus, III.2.39-46)

Rappelons que Platon ne dévalorise pas le langage des gestes lorsqu'il se substitue à la voix devenue muette naturellement :

Cratyle. Si nous n'avions point de voix et que nous voulussions nous montrer les uns aux autres, n'essaierions-nous pas comme le font les muets de les indiquer avec les mains, la tête et le reste du corps.

(Cratyle, 422b)

La page dans L'Apologie de Raimond Sebond à la gloire du langage des gestes est mémorable ${ }^{8}$. Pour Cicéron dans le De Oratore, la gestuelle est censée refléter les mouvements de l'âme (De Oratore, III.216).

Cicéron accuse Socrate d'avoir séparé la langue et le cœur (De Oratore, III.XVI.61). Avant lui la connaissance et la pratique des notions n'avaient qu'un nom et c'était la philosophie. L'éloquence était au nombre des principales vertus (III.XV.55). Désormais la suspicion s'attache à la langue. Il faut donc s'appliquer à renouer action et parole (III.XVI.59). The Murder of Gonzago donne une voix au dumb show, l'ouvre au sens et à la vérité, en reliant à nouveau mot et gestuelle.

Il est remarquable que ce soit dans une pièce d'inspiration platonicienne et hermétique comme Hamlet que l'on trouve la louange de l'acteur comme l'être le plus susceptible de relier «the action to the word, the word to the action» (III.2.16-7; 128) selon l'idéal cicéronien. (Cicéron fait une distinction entre la gestuelle qui traduit les paroles comme au théâtre et celle qui éclaire l'idée et la pensée et on retrouve chez lui la même admiration pour Roscius (sans doute empruntée aux Stoïciens) que celle de Hamlet (Hamlet, II.2.285)). Hamlet s'inscrit dans une réflexion sur le stö̈cisme et si la pièce est une critique de ses aspects les plus controversés à la Renaissance, Shakespeare partage néanmoins avec les Stö̈ciens le désir de réhabiliter l'acteur ${ }^{9}$.

Hamlet, imitateur de la folie et donc suspect, ou Hamlet en acteur parfait comme Roscius établissant la plus harmonieuse unité 
entre le geste et la voix ? Hamlet du côté de Platon ou de Cicéron ? La question est centrale à la pièce et reflète le débat entre deux formes d'humanisme, un humanisme cicéronien ou un humanisme platonicien.

\section{La Voix Réifiée}

Ainsi qu'on vient de le voir dans Hamlet, la voix n'est pas toujours du côté du langage. Comme la voix du fantôme, elle peut appartenir à l'univers des choses, naître de l'univers, se confondre avec les murmures des vagues de la mer ou des coups de tonnerre. Elle n'est après tout que l'un des quatre éléments. Tout entière faite d'air elle est ce qui reste d'une nymphe amoureuse dont le destin était de répéter les mots de celui qui ne l'aimait pas : selon Ovide, d'Écho il ne subsiste que la voix et les os transformés en pierres (Métamorphoses, III) ${ }^{10}$. Elle s'enticha de Narcisse qui dans les emblèmes d'Alciat représente le vice Philautia ${ }^{11}$, l'amour coupable de soi qui, nous le verrons, se confond avec le silence suspect. C'est la voix d'Écho qui fait résonner la maison de Rumeur dont la charpente en bronze «is full of noises, repeating words and giving back the sounds it hears» (Métamorphoses, XII). La rumeur couverte de langues chevauche le vent dans l'induction de 2 Henry IV, 4. Dans Richard III, la conscience la mimera comme pour punir le tyran d'avoir trop abusé d'une éloquence perverse et se couvrira de mille langues :

My conscience hath a thousand several tongues And every tongue brings in a several tale And every tale condemns me for a villain.

(Richard III, V.3.193)

Ces images d'une voix vagabonde, corporelle, plurielle illustrent son caractère suspect. La querelle des Universaux avait contribué à déconnecter un peu plus encore la voix d'avec sa parenté avec le Verbe divin. Roscelin, le premier nominaliste médiéval (1050-1124) réduit les Universaux à n'être que des mots définis comme flatus vocis, c'est ce que l'on appellera le vocalisme $^{12}$. Mais craignant que l'aspect corporel de la voix - ce que Montaigne appelle «ce corps aéré de la voix» (Les Essais, II.VI, p. 359) - ne contamine de sa choséité l'Universel, Abelard (10791142) sépare dans le mot la voix, réduite toujours qu'à n'être une simple création naturelle, une res (comme la pierre dans une statue est une res) et le sermo, la part signifiante du mot qui elle seule 
peut être reliée à l'Universel (comme la forme que l'homme donne à la pierre pour qu'elle devienne statue ${ }^{13}$. Ces subtilités de la scolastique médiévale éclairent la scène du balcon dans Romeo and Juliet où la voix réifiée préside au faux dialogue entre les deux amants. Les mots qui s'y disent ne sont que des sons vocaux sans signification et se répondent comme des échos, comme si Narcisse et Écho répétaient à Vérone leur idylle impossible.

Rom. She speaks yet she says nothing. What of that ?

Her eye discourses, I will answer it.

I am too bold. 'Tis not to me she speaks.

(Romeo and Juliet, II.2.12-4)

C'est à Écho que se voue Juliet :

I would tear the cave where Echo lies

And make her airy tongue more hoarse than mine

With repetition of my Romeo's name.

(Romeo and Juliet, II.2.162)

Ce nom dans la bouche d'Écho n'est qu'un flatus vocis. Il n'est guère surprenant que la question nominaliste de Juliet, "What's in a name ?» se pose tout de suite après ce faux dialogue qui ressemble si étrangement au langage d'Ophélie. Romeo ne doit-il pas «botch the words up to fit [his] own thoughts» (Hamlet, IV.5.10) ? Les mots échos sont aussi arbitraires que les noms qui selon Montaigne «acquièrent de la fierté et de la magnificence à les ouïr seulement sonner» (Les Essais, I.XLVI.267a) ${ }^{14}$. Shakespeare vocalise les noms, devient nominaliste pour dénoncer la vanité et le faux-semblant des hommes qui selon Montaigne «ne s'enflent que de vent et se manient à bonds comme les balons» (Les Essais, III.XII.104b).

A chaque fois que le mot se détache de son fondement ontologique il se répète, il s'enfle d'air, devient écho. Dans Love's Labour's Lost, Mote, le bien nommé, joue le rôle d'Écho dans les faux dialogues entre les amants et est renvoyé lorsqu'il a assez fait preuve de son inanité.

On peut ajouter l'autre exemple célèbre de Richard $I I$, pièce contemporaine de Romeo and Juliet, où Exton fera résonner deux fois la petite phrase d'Henry IV, né de rien, né du vent. 
Have I no friend? Quoth he. He speaks it twice And urg'd it twice together did he not?

( Richard II, V.4.3-5)

Un acte maudit naîtra de ces voix comme dans Romeo and Juliet, «three civil brawls» étaient nées de «an airy word» (Romeo and Juliet, I.1.87).

Shakespeare joue avec l'aspect vocal des mots, les fait rebondir comme des effets d'échos ou les creuse pour les vider de leur substance. La voix suspecte rend le mot suspect, le réduit à l'état de bruit. C'est le sens de ce que l'on peut comparer à l'épisode des paroles gelées de Rabelais dans All's Well that Ends Well où les syllabes insignifiantes ne sont que des échos de vocalises creuses proches des cris des choucas («chough's language») ironiquement qualifiées de langage (All's Well that Ends Well, IV.1.19). Ne s'entend plus alors que le «sleepy language» de l'île de Prospero et maudit alors celui qui trouverait «meaning in his snores» (The Tempest, II.1.215). Car alors il ferait résonner des bruits sataniques, les «roaring voices» de King Lear (II.3.14) «venant d'affre», de cavités corporelles grossières, non prévues par l'artisan divin pour un tel usage.

Si Shakespeare joue de ces possibilités du langage, il ne se laisse guère fasciner par les transgressions vocales et toujours il maintient l'opposition entre une voix porteuse de vérité reliée au Logos et la voix creuse du mensonge. Ainsi Ferdinand oppose clairement ces deux langages :

If I speak true, if hollowly...

(The Tempest, III.1.70)

\section{La voix juste}

Et pourtant la voix, malgré sa corporalité peut échapper à son caractère suspect. Comme s'il voulait tester jusqu'à leurs extrêmes limites les capacités de la voix à véhiculer la vérité, Shakespeare lui fait subir les expérimentations que la scolastique faisait subir au nom pour comprendre de quelle manière il est relié aux choses. Shakespeare va jouer avec la voix comme on peut jouer avec le nom. Il s'agit de vérifier l'hypothèse selon laquelle il y a un rapport de justesse, de vérité entre l'individu et sa voix. La voix peut-elle être le sceau confirmant l'identité de l'individu ? Jessica reconnaît Lorenzo à sa voix (The Merchant of Venice, II.6.27). De même 
Romeo :

My ears have not yet drunk a hundred words

Of thy tongue's uttering, yet I know the sound.

(Romeo and Juliet, II.2.58-9)

Cela suffit-il à fonder l'authenticité de la voix ? De nombreux cas de figure, des questions expérimentales vont bousculer cette correspondance, pour la pousser à bout, la vérifier. De même que l'homonymie et la synonymie mettent en valeur l'arbitraire du signe $^{15}$, de même certains cas où la voix entretient des rapports extrêmes avec l'individu permettent de troubler les repères vocaux de l'identité.

Parfois une seule voix se réfère à deux personnes : c'est le cas où la voix de Viola et celle de Sebastian ne font qu'un.

One face, one voice, one habit and two persons.

(Twelfth Night, IV.1.213)

Dans The Tempest, Stephano croit entendre la voix de Trinculo mais c'est Ariel qui parle (The Tempest, III.2.40-80). Ici comme dans Twelfth Night une voix se réfère à deux personnes et cet effet est produit par le don mimétique d'Ariel alors que Viola n'imite pas celle de Sebastian, leurs voix étant réellement identiques et symbolisant leur harmonie divine.

Dans un deuxième cas, deux voix différentes correspondent à une seule personne. C'est la voix d'Edgar avant et après la scène de la falaise dans King Lear. "Thy voice is altered» (King Lear, IV.6.7) dit Gloucester à celui qu'il croit être Poor Tom mais qui est en train de redevenir Edgar. Ici s'il y a double voix, c'est qu'Edgar a imité Tom o' Bedlam. La voix ainsi si facilement déguisée n'est-elle pas de nature trompeuse? Et même si elle est présentée comme une preuve de vie au même titre que le mouvement dans la scène de la statue de The Winter's Tale, elle ne peut être garante à elle seule de vérité. Le sens de la scène finale de The Winter's Tale est de démontrer que toutes les expérimentations empiriques ou magiques que l'on fera subir à la voix ne sont rien à côté du miracle que constitue l'investissement de la voix humaine par la vérité. Il ne $s$ 'agit pas de faire parler Hermione selon un rituel magique que saint Augustin réprouvait lorsqu'il parlait des statues animées d'Asclepios ${ }^{16}$. On ne reconnaîtra pas Hermione à sa voix mais à sa 
capacité d'entrer dans un circuit de communication avec Leontes et Perdita. C'est ce que souligne Paulina qui à la demande de Leontes de faire parler la statue enjoint à ce dernier de prendre la parole.

I like your silence, it the more shows off

Your wonder : but yet speak ; first you, my liege...

(The Winter's Tale, V.3.21-2)

Et c'est en réponse à la prière de Perdita que la statue-Hermione exprimera ses premières paroles (V.3.121-8). C'est aussi ce que soulignent les paroles de Jessica qui demande à Lorenzo d'investir sa voix de vérité :

Who are you? Tell me for more certainty

Albeit I'll swear that I do know your tongue.

(The Merchant of Venice, II.6.26-7)

La voix ne peut être porteuse d'authenticité que dans l'expérience intercommunicative. Dans cette analyse sur le pouvoir distinctif de la voix Shakespeare s'inspire peut-être aussi de la problématique des deux Cratyles. Même si on imaginait une copie absolument parfaite de Cratyle, même si on y ajoutait «les caractères de mollesse et de chaleur, y mettant le mouvement, l'âme et la pensée...» (Cratyle, 432b-c) on pourrait encore distinguer le vrai Cratyle du faux Cratyle. Socrate réaffirme ainsi que la vérité transcende les preuves que l'on peut en apporter, Paulina dira : «It is requir'd / You do awake your faith» (The Winter's Tale, V.3.94-5). Paulina la bien nommée, si on en fait un porte-parole de saint Paul et de saint Augustin, dit ici qu'au-delà de la matérialité vérifiable de la voix, c'est le Logos qu'elle véhicule qui est garant de la vérité.

\section{La voix assourdie ou le déni du présent}

La voix peut être du côté des choses, des res mais elle est aussi du côté du langage, des verba. Alors, elle cesse d'être un bruit ou un écho et elle épouse le mouvement même de l'âme. Elle ne trompe plus, elle cesse d'être suspecte et devient alors suspect celui qui ne l'entend plus, ne la reconnaît plus. La voix se fait musique, expression de la dignité même de l'individu qui seul a le pouvoir de la faire parler et de se relier ainsi à l'harmonie de l'univers. 
How unworthy a thing you make of me... You would play upon me... and there is much music, excellent voice, in this little organ, yet you cannot make it speak.

$\left(\right.$ Hamlet, III.2.345-51) ${ }^{17}$

Saint Augustin s'émerveillait de la voix humaine : «ne trouves-tu pas merveilleux ce qui a lieu au sujet de nos paroles, du son de notre voix, choses d'un instant?» (Lettre, 137.2.7-8). Cet émerveillement réussit jusqu'à lui faire oublier que son aspect corporel pouvait parfois «salir» comme il le dit dans le De Ordine les nombres et la raison qui fondent la musique. Dans un célèbre développement du livre XI des Confessions, saint Augustin se sert de la voix pour illustrer sa théorie du temps: la conscience du temps présent se mesure comme on mesure les syllabes de la voix humaine. Cette conscience du temps démontrée par le miracle de la voix met l'individu en harmonie avec le monde. En effet, ce qui n'est pas soumis à la loi du temps est informe (Les Confessions, XII. ch. 12). Frank Kermode avait établi les correspondances entre l'analyse augustinienne du temps et Macbeth dans The Sense of an Ending ${ }^{18}$. Cette étude sur la voix permet d'établir d'autres correspondances entre saint Augustin et Shakespeare dans l'espace de cette pièce. On remarque par exemple que Shakespeare reprend la même métaphore de la voix humaine pour exprimer la division en unités de temps. "The last syllable of recorded time» (Macbeth, V.5.21) fait écho aux huit syllabes du Deus creator omnium (Les Confessions, XI. ch. 27) qui servent d'exemple à la démonstration augustinienne soulignant que la loi du temps est celle qui donne à la voix humaine sa légitimité. Mais Macbeth ne peut plus mesurer le temps qu'à l'aune de l'avenir dans les syllabes qui composent la triple succession des «tomorrow» (Macbeth, V.5.19-21), véritable déni de la triple décomposition augustinienne du présent en «présent du passé, présent du présent, présent du futur» (Les Confessions, XI. ch. 20), comme si le mal pouvait se définir comme un envahissement dans la conscience d'un futur destructeur de présent.

Entendre la voix c'est entrer dans la logique du temps qui n'est toujours qu'une modalité du présent parfaitement représentée dans l'immédiateté de la sensation et de la trace mémorisée produite par la voix. Mais Macbeth désaccorde cette harmonie. Il n'entend plus dans la voix humaine ce qui en fonde la mesure. Il n'entend plus la voix humaine dont la prise de conscience lui permettrait d'être de plain-pied avec l'immédiateté du présent. C'est ainsi que 
Shakespeare s'inspirant de saint Augustin illustre chez Macbeth son déni du temps présent, caractéristique selon Plutarque et Montaigne d'un stoïcisme que la Renaissance réprouvait.

A deux reprises avant le meurtre de Duncan, Macbeth avait proposé à Banquo de parler avec lui dans l'intérim - cet espace de temps qui sépare l'intention d'un acte de sa réalisation (Macbeth, I.4.154-6 \& II.1.22-4) jamais ce dialogue pris au temps n'aura lieu comme s'il n'y avait jamais assez de présent pour pouvoir parler dans la fatale fuite en avant dans laquelle Macbeth s'engage désormais. En détruisant sa conscience du présent Macbeth se condamne à un isolement peuplé de bruits qu'il ne peut plus désormais reconnaître comme les mots signifiants du langage. Après le meurtre de Duncan, Macbeth n'entend plus sa propre voix comme en témoigne le court dialogue avec Lady Macbeth. «I have done the deed. Didst thou not hear a noise ?» (Macbeth, II.2.14) ce bruit ce n'est ni le hululement de la chouette ni le cri du grillon, mais la voix de Macbeth. C'est comme si l'empreinte de sa propre voix ne pouvait plus rester gravée dans sa mémoire.

L. Macb. Did not you speak? Macb. L. Macb. Macb. When? As I descended? L. Macb. Ay. Macb. Hark! Now.

(Macbeth, II.2.16-8)

Et ce «Hark !» annonce le début des hallucinations auditives de Macbeth qui désormais ne va plus entendre que des voix intérieures déliées du temps présent.

Partout dans le royaume la voix humaine résonne et Macbeth est le seul à ne pas l'entendre :

Each new morn,

New widows howl, new orphans cry, new sorrows

Strike heaven on the face, that it resounds

As if it felt with Scotland, and yell'd out

Like syllable of dolour.

(Macbeth, IV.3.4-8)

La voûtè du ciel est un tympan qui devient bouche hurlante d'où sortent les syllabes de la douleur, c'est à dire la voix humaine 
découpée en unités mesurables. Le ciel intériorise la voix humaine comme Macbeth ne sait plus le faire et permet qu'elle y laisse son empreinte pour que s'en constitue la mémoire. En Écosse, le tyran laisse hurler la voix qui passe inaperçue puisque est occultée la conscience du présent.

Sighs, and groans and shrieks that rent the air Are made, not mark'd ; where violent sorrow seems A violent ecstasy.

(Macbeth, IV.3.168-70)

Pourtant les cris se rapprochent du château de Dunsinane. Et ces cris sont au présent. «The cry is still "they come"» (Macbeth, V.5.2). Et voilà que le cri pénètre dans le château. La didascalie annonce, «a cry within, of women» et encore une fois pour Macbeth ce cri n'est qu'un bruit comme n'avait été qu'un bruit sa propre voix lorsqu'il descendait de la chambre de Duncan: «What is that noise?» (Macbeth, V.5.7) avait-il demandé. Et ce cri comme le précédent est aussi au présent : «It is the cry of women, my good lord» (Macbeth, V.5.8). Il annonce la mort de la reine dont le déni par Macbeth représente le déni du présent.

L'influence de saint Augustin, qui était professeur d'éloquence et avait eu une formation de rhéteur ${ }^{19}$, sur l'importance donnée à l'humanisme naissant est souvent passée sous silence car on a l'habitude de voir chez lui l'instigateur de courants plus sévères comme le jansénisme. Jean Delumeau rappelle qu'il fut «un réveilleur de l'humanisme» ${ }^{20}$. Alastair Hamilton voit en lui «a Christian counterpart to Cicero» ${ }^{21}$, et on le verra grand favori de l'époque baroque ${ }^{22}$. Si l'humanisme fait l'apologie de la vita activa contre la vita contemplativa, on peut être étonné que ce grand contemplatif ait défendu les valeurs qui seront celles de l'humanisme. Pourtant, grand lecteur de Cicéron, saint Augustin pensait que l'éloquence, l'art d'user de sa voix, était la servante inséparable de la charité dans le De Doctrina christiana ${ }^{23}$.

\section{«The silent court» : la cour du roi de Navarre et la cour du roi Lear}

Saint Augustin est un allié de Shakespeare dans son combat contre un certain silence. En effet, de quel silence s'agit-il? Comment situer l'œuvre de Shakespeare entre le silence des obscuri viri de l'ancienne scolastique averroïste qui prônait le retrait de la vie civile et le silence de la vie contemplative, et les nouveaux 
humanistes qui en réaction à un certain aristotélisme remettaient Platon au goût du jour et comme Pic de la Mirandole ou les humanistes pétrarquisants, cherchaient à revaloriser le silence ${ }^{24}$. Contre le silence des premiers, objets constants du ridicule de Rabelais, Shakespeare écrit Love's Labour's Lost où il dénonce l'abstinence de la parole, symbole de l'abstinence sexuelle. Si une femme s'approchait de la cour du roi de Navarre elle serait menacée de perdre sa langue (Love's Labour's Lost, I.1.122). Le roi de Navarre veut instaurer «a silent court» (Love's Labour's Lost, II.1.24). Rien n'y est plus suspect que la voix. A la cour sévit Holofernes, pur produit des facultés des arts, «an artsman». Maître des arts des facultés parisiennes, il est l'un de ces hommes obscurs dont Rabelais se moquait (Love's Labour's Lost, V.1.75). Il enseigne dans le collège de Montaigu que l'un de ses plus célèbres élèves, Érasme, appela «le collège vinaigre» ${ }^{25}$.

Do you not educate youth at the

Charge house on the top of the mountain?

(Love's Labour's Lost, V.1.75)

demande Armado, soulignant la localisation de ce collège sur la montagne Sainte Geneviève à Paris. L'association de la cour du roi de Navarre avec ce collège n' est pas un hasard. D'abord tenu par les scotistes, il devint un haut lieu du renouveau nominaliste sous Jean Mair qui y enseigna à l'époque d'Érasme. Le collège de Montaigu raconté par Érasme c'est l'institut Lowood raconté par Charlotte Brontë. On y meurt, on y perd la santé ${ }^{26}$. Le fantôme de Duns Scot (1265-1308) hante les lieux. Ce franciscain prônait un retrait de la vie de la cité justifié par sa théorie de la personne définie comme «ultima solitudo» 27 . Comme 1'homme ne doit pas dépendre des autres, Scot ne considérait pas le mariage comme une institution de droit naturel. Berowne semble faire allusion à ce débat lorsqu'il dit «we cannot cross the cause why we were born» (Love's Labour's Lost, IV.3.216). Les nominalistes, bien qu'opposés sur de nombreux points à Duns Scot représentant de la via antiqua, partageaient avec lui certains aspects de cette théorie de la personne. Ockham (12801348) pense que la personne est «per se subsistens» ${ }^{28}$. L'individualisme nominaliste pourrait avoir des conséquences graves sur la vie de la cité et s'oppose au thomisme qui favorisait la vita activa : saint Thomas croyait en une nature humaine commune à tous. Cette pensée est résumée par l'un de ses disciples Pierre d'Auriole: «natura enim nostra est politica et civilis et 
communicativa» ${ }^{29}$. Le propre de l'homme est donc de parler.

Dans Love's Labour's Lost, Shakespeare tranche ici en faveur de la voix, comme Érasme, comme Rabelais. Déguisés en moscovites (Love's Labour's Lost, V.2.266) le roi, Dumaine, Longaville et Berowne dialoguent avec les trois femmes mais les paroles semblent gelées comme dans le texte de Rabelais ou de Castiglione dont on trouve aussi un écho dans All's Well that Ends Well. Lorsque le masque commence, les mots des dialogues riment, se font écho, badinent mais ne parlent pas. La voix qui sort des masques est suspecte et guère meilleure que le silence qu'elle cherche à conjurer. L'un de ces masques est d'ailleurs celui du silence. Il s'agit de celui de Longaville:

Katharine. What, was your visor made without a tongue?

(Love's Labour's Lost, V.2.242)

Katharine parle aux deux niveaux littéral et figuré car l'une des particularités de ces masques est qu'ils étaient maintenus par une tige en verre appelée «tongue» que l'on serrait entre les dents. Cette langue-là empêchait la parole. Longaville parle de «my speechless vison» (Love's Labour's Lost, V.2.246). Longaville et ses amis devront enlever un tel masque du silence et pour apprendre à parler devront tenter de communiquer avec le vrai silence des «speechless sick» (Love's Labour's Lost, V.2.840).

La cour de Lear est la maison de la Rumeur où les complots naissent des mots. A l'inverse, le silence de Cordelia porte en luimême l'éloquence du fou dont le flot de parole naît des sources d'une sagesse hermétique et énigmatique confrontée sans cesse à la modernité machiavélienne. Ainsi les deux cours, celle du roi de Navarre et celle du roi Lear illustrent deux types de silence et en problématisent les enjeux.

Érasme mettait la viva vox du côté de la vie :

Viva vox the living word, was the term used in old times for anything not written, but taken straight from the mouth of the speaker, lifelike as it were and effectual... writing is... a kind of voice - mimicking the real voice, gesture and movement are lacking ; in a word, life. ${ }^{30}$ 
Mais Shakespeare module cet avis et malgré sa prise de position «érasmienne» contre le silence des hypocrites, il prend le temps de faire la différence entre deux types de silence et réaffirme sa position platonicienne et hermétique aussi clairement exprimée dans Hamlet. Shakespeare émet des réserves sur l'opportunité de remplacer le mot écrit par la viva vox. C'est le fameux passage de Twelfth Night où Feste préfère lire oralement la lettre de Malvolio pour que l'intonation de sa voix aide sa maîtresse à mieux se persuader de la folie de son intendant. «You must allow vox» (Twelfth Night, V.1.295). Olivia craint cette voix suspecte et demande à Fabian de lire la lettre pour plus de vérité. De même sir Toby préfère ne pas donner la lettre de Sir Andrew à Viola car si elle la lisait, elle comprendrait la vérité. La voix vox aidera Sir Toby à tromper son monde : «I will deliver his challenge by word of mouth» (Twelfth Night, III.4.192). Cette valorisation de la lecture silencieuse considérée comme porteuse de plus de vérité convient à une pièce qui peut se résumer à la quête d'une sagesse hermétique dont on peut lire entre les lignes les signes caractéristiques ${ }^{31}$. Mais l'histoire de la lecture silencieuse permet aussi de comprendre qu'elle fut une arme efficace pour la propagation de la Réforme qui encourageait la lecture individuelle et solitaire de la Bible ${ }^{32}$. La voix serait a contrario le véhicule des interprétations forcées du dogmatisme, du fanatisme religieux des inquisiteurs romains ou des puritains tous deux caricaturés par le clown déguisé en sir Topas.

\section{Lady Silence and Lady Tongue}

Malgré les dangers que représentent l'utilisation de la voix porteuse des passions du corps (1 Henry VI, IV.1.182), la cité se fonde sur la réalité d'une nature humaine «communicative». La cité avec l'humanisme, devient une cité-parlement qui valorise la vita activa sans exclure chez Shakespeare comme chez saint Augustin la vita contemplativa. Vickers fait de Shakespeare le pourfendeur des valeurs du silence et du repli sur soi coupable ${ }^{33}$. Mais il est important de rajouter que la position de Shakespeare est tempérée par un platonisme qui lui empêche d'oublier les vertus du silence sans pour autant le confondre avec celui des obscuri viri. Dans la cité de Shakespeare deux allégories s'affrontent: Lady Silence et Lady Tongue. Dans sa traduction de la vie de Numa par Plutarque (1595) North traduisit par Lady Silence le nom de la muse Tacita qui aurait aidé le second roi légendaire de Rome à fonder la cité selon des préceptes pacifiques et silencieux ${ }^{34}$. La Lady Tongue de $M u c h$ Ado about Nothing serait son double ironique. Les débats de ces deux 
femmes s'éternisent dans les pièces politiques. Ils reflètent les positions contradictoires des commentateurs d'Aristote dont les livres de philosophie sociale commencèrent à paraître entre 1260 et 1270. La lecture averroïste d'Aristote choquait puisqu'elle prétendait que l'amour de soi narcissique Philautia pouvait dépasser l'amour de Dieu ou de la patrie ${ }^{35}$. Pour les averroïstes, l'idée du sacrifice individuel au bien commun débattue dans Henry $V$, devenait suspecte et le philosophe citoyen était encouragé de ce fait à l'isolement et au silence de la vita contemplativa. Cette position est discutée dans Richard II qui plus que tout autre représente cet individualisme hautain d'origine averroïste qui fait pencher la balance en faveur de Bolingbroke.

In your lord's scale is nothing but himself

But in the balance of great Bolingbroke

Besides himself are all the English peers.

(Richard II, III.2.85-7)

Coriolan représente ce même isolement :

As if a man were author of himself

And knew no other kin.

(Coriolanus, V.3.34-7)

Ces deux hommes dédaignent: «The courtship of the common people» (Richard II, I.4.19). Mais si Philautia semble condamnée comme un repli sur soi narcissique dans Richard $I I$, le scepticisme engendré par la critique plutarquienne du stoïcisme oblige à affiner la démonstration et fait dire à la duchesse d'York dans Richard II que l'amour de soi est une valeur primordiale : «Love loving not itself none other can» (Richard II, V.3.86), thème que l'on retrouve dans 1 Henry IV (II.3.100-1).

Shakespeare fait apparaître sur la palette de ses pièces politiques les nuances du dialogue entre Lady Silence et Lady Tongue. D'un côté le noble silence de Cordelia se distingue du silence suspect de Coriolan tandis que d'un autre, la voix du peuple n'est pas toujours porteuse de bon sens et peut se comparer à un aboiement de chien (Coriolanus, II.3.20). La vox populi peut en effet être équivoque et bestiale. Dans son essai De la Gloire, Montaigne rapporte cette phrase de Demetrius qui «disoit plaisamment de la voix du peuple qu'il ne faisoit non plus de recette de celle qui lui 
sortoit par en haut que de celle qui lui sortoit par en bas» (607c), source sans doute de ce monstre délicat découvert par Stephano dans The Tempest : «his forward voice is now to speak well of his friend, his backward voice is to utter foul speeches and to detract» (The Tempest, II.2.85-90). La vox populi est une de ces voix qui miment l'animalité. Comment la faire participer au banquet politique ? Cette question centrale à Coriolanus est celle que se pose l'Angleterre élisabethaine comme le suggère P. Brockbank, dans sa préface à Coriolanus ${ }^{36}$ lorsqu'il cite sir Thomas Smith dans De Republica Anglorum (1583) où le peuple dialoguant avec le roi fait de la cité une cité-parlement.

Coriolan oppose au «din confused» (Coriolanus, III.3.20) de la vox populi sa propre voix qu'il considère libre comme la prière :

A voice as free

As I do pray the gods.

(Coriolanus, III.3.73)

Cette liberté de la parole ne doit pourtant pas être sanctifiée et mise sur le même plan que la prière. Elle s'oppose au contraire à ce subtil pouvoir de la parole qui enchaîne la langue de l'orateur aux oreilles de ceux qui l'écoutent. L'image est celle d'Hercule qui vainquit ses ennemis non pas par les armes mais par les lettres comme le décrit l'un des emblèmes d'Alciat, «Éloquence est plus excellente que force» ${ }^{37}$. Sur l'emblème apparaissent les chaînes qui percent la langue d'Hercule et la relient aux oreilles de ses ennemis subjugués. Comme la plupart des héros shakespeariens Coriolan ne comprend pas cette morale des emblèmes et confond la véritable force avec celle des armes concluant que l'éloquence est affaire de femmes:

My throat of war be turned Which choired with my drum into a pipe Small as an eunuch or the virgin voice That babies lulls asleep.

(Coriolanus, III.2.115)

Coriolan n'est donc guère meilleur que Parolles qui selon Lafeu «is a good drum but a naughty orator» (All's Well that Ends Well, V.3.249). A contrario la véritable force, la seule force physique justifiée est celle qui peut se confondre avec le pouvoir de la voix. A la force pulsionnelle et bestiale de Macbeth s'oppose la force 
juste qui fait dire à Macduff «my voice is in my sword» (Macbeth, V.8.7).

Il n'est guère surprenant alors de constater chez Shakespeare que la femme toujours soit la gardienne d'un Logos qui s'oppose au silence arrogant des fanfarons belliqueux. L'éloquence donne à la femme une force herculéenne qui lui permet de tenir tête à l'ennemi. Mais c'est plutôt parce que la faiblesse physique de la femme sert de faire-valoir à la force de la parole que la femme est choisie pour représenter l'Éloquence dans l'œuvre de Shakespeare. L'éloquence est d'autant plus forte qu'elle se sert d'organes considérés comme dérisoires, le corps de la femme ou, dans l'exemple cité plus haut une mâchoire d'âne. Dans All's Well that Ends Well le roi dit à Helena en un distique rimé :

Methinks in thee some blessed spirit doth speak

His powerful sound within an organ weak.

(All's Well that Ends Well, II.1.175)

Dans Coriolanus ce sont deux femmes qui personnifient la vraie force: Volumnia, pour qui «action is eloquence» (Coriolanus, III.2.75) et Virgilia symbole d'un «gracious silence» (Coriolanus, II.1.174) qui en est le double et qui se démarque du silence orgueilleux du héros. Volumnia ne tient-elle pas d'Hermes, le dieu du silence comme de l'éloquence, le secret de sa force ? Alciat décrit comment Mercure-Hermes donna à Ulysse la fleur nommée Moly, «contrepoison aulx breuuages Circes» ${ }^{38}$, symbole de l'éloquence car elle «ha noire racine, fleur blanche et purpurine» comme l'éloquence «au commencement obscure» et qui devient «florissante, claire et honorée». Volumnia tel Ulysse dans Troilus and Cressida n'hésite pas à user de tous les tours et détours de la rhétorique :

\section{Speak}

But with such words that are but roted in Your tongue, though bastards and syllables Of no allowance to your bosom's truth.

(Coriolanus, III.2.55)

Les héroïnes de Shakespeare sont les «nourrices» de la parole (Richard II, V.3.111). Nous avons vu que la duchesse dans Richard II veut apprendre l'art de la parole au nouveau roi pour qu'il ne tombe 
pas dans les travers du précédent, victime de ces «duels de mots» qui furent sa perte. L'éloquence de Portia dans The Merchant of Venice s'oppose au silence suspect de Shylock qui ne cesse de répéter «I'll have no speaking, I will have my bond» (The Merchant of Venice, III.3.12-3). Isabella dans Measure for Measure quitte le silence du cloître pour combattre la tyrannie au cœur même de la cité, et Rosalind, dans le silence de la forêt emprisonne ses interlocuteurs dans les chaînes de l'éloquence. Dans le De Vulgari Eloquentia, Dante était choqué par l'épisode des Écritures qui rapporte que la femme fut la première à parler : «Imaginer qu'un acte aussi glorieux du genre humain n'ait pas pris source dans l'homme plutôt que dans la femme, c'est inconvenance» ${ }^{39}$. Certes, en faisant jouer à ses héroïnes le rôle noble des porte-parole de la vérité, Shakespeare pourrait sembler moins misogyne que Dante. Mais ce qu'il cherche plutôt à prouver, c'est la force du Logos qui peut emprunter des organes de la parole aussi dérisoires que ceux d'une femme.

L'organe de la parole chez la femme reste suspect. Une gravure de A. Woensam (c. 1525) montre le portrait d'une «femme avisée» dont la bouche est close par un cadenas. Malgré les apparences, cette allégorie pourrait bien représenter les nobles héroïnes de Shakespeare dont l'Éloquence n'est que le fruit de la sagesse biblique. Bardée de symboles diaboliques inversés, comme le serpent qui signifie Prudence ${ }^{40}$, la femme éminemment suspecte ne peut toujours pas laisser entendre sa voix corporelle et les grandes figures de la féminité shakespearienne sont représentées sur scène par un corps masculin imparfait - puisque enfant encore et non parvenu à maturité. La mauvaise réputation de Lady Tongue est toujours florissante.

\section{«A speechless song» (sonnet 8)}

The Tempest décrit la genèse de la voix humaine, ce moment où elle n'est pas encore encombrée des mots du langage mais où elle participe comme son parmi d'autres sons à l'univers sonique des matins du monde - Caliban nous décrit certains d'entre eux : «Sounds and sweet airs that give delight and hurt not» (The Tempest, III.2.133-41) - des sons qui charmeront Fellini dans $L a$ Dolce Vita. Ces voix sans mots qui bercent Caliban enchantent aussi Alonso qui les qualifie d' «excellent dumb dicourse» (The Tempest, III.2.39) et en fait ainsi l'équivalent à de la «speechless song» du sonnet 8 , l'harmonie la plus pure de la musique polyphonique naissante, «the true concord of well tuned sounds» (sonnet 8 ). Cette 


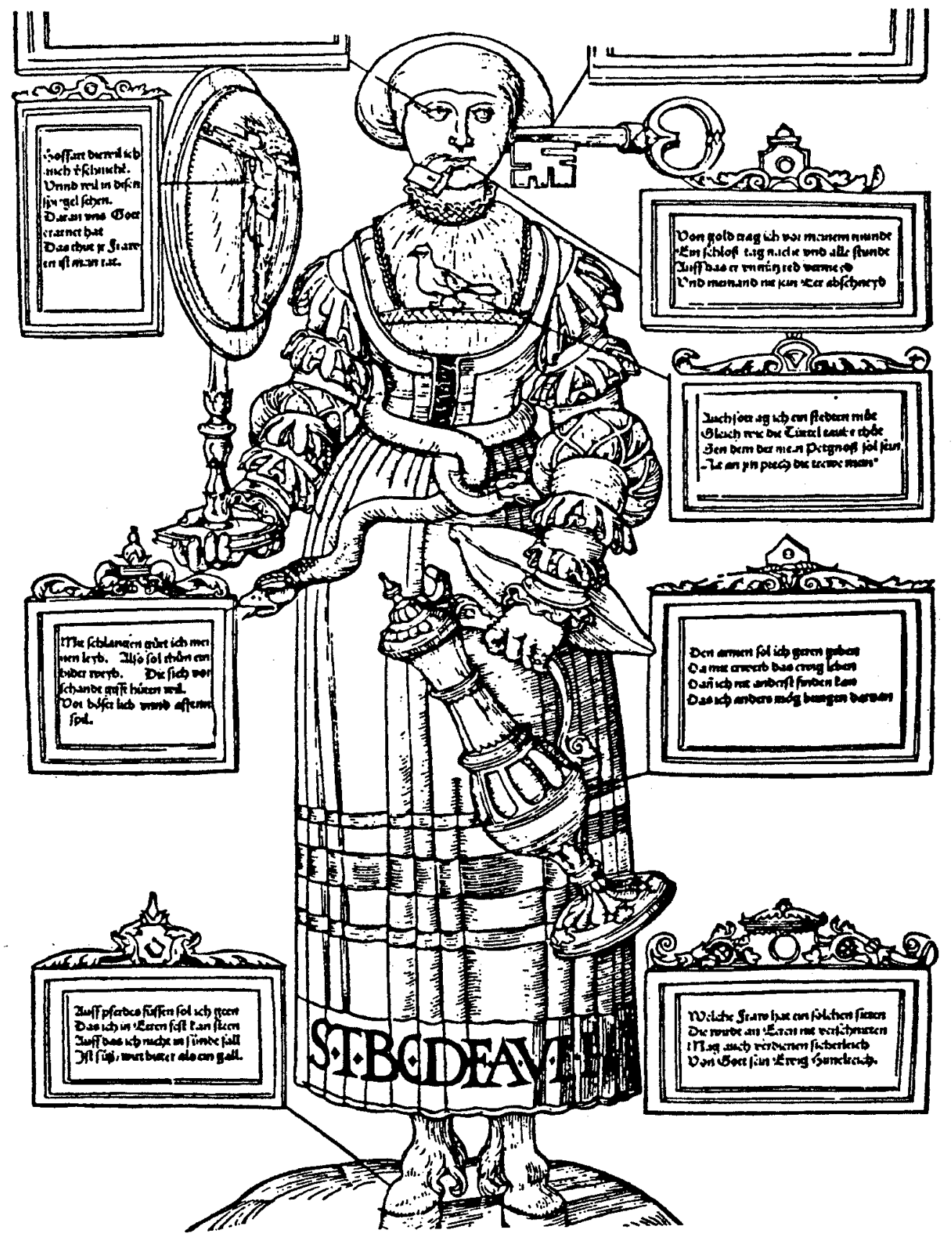

A. Woensam, La femme bien avisée (c. 1525) 
valorisation de la voix, traversée par le langage, semble contredire le discours platonicien, si bien que l'on se demande, et c'est suggéré par Stephen Greenblatt, s'il n'y a pas une remise en cause de la laus eloquentiae que Cicéron voyait comme la force civilisatrice qui fait passer les hommes d'une vie barbare à la société civilisée. Il y aurait une nature que le langage dénature :

\section{My father's of a better nature sir}

Than he appears by speech.

(The Tempest, I.2.497-8)

Après avoir appris le langage à Caliban (The Tempest, 1.2.362), Prospero ne rêve-t-il pas qu'à le faire rugir, à le faire revenir à l'état de cette «thing most brutish» (The Tempest, I.2.356) ? Gonzalo, ce missionnaire de la civilisation, abuse du langage: il est «a spendthrift of his tongue» au point d'être en passe de devenir corbeau (The Tempest, II.1.24-9). Cet «excellent dumb discourse» serait selon Greenblatt le langage de l'homme sauvage du moyen âge décrit dans The Faerie Queene comme :

A soft murmur and confused sound

Of senseless words, which Nature did him speak

(The Faerie Queene, VI.4.2)

Il viendrait contredire la bulle du Pape Paul III de 1537 selon laquelle les Indiens n'étaient que des bêtes muettes bonnes pour $l^{\prime}$ esclavage ${ }^{41}$. Sans compter que l'on remet en cause le mutisme des bêtes: L'Apologie de Raimond Sebond bouscule les frontières entre les espèces et prétend que : «les animaux ont un discours au-dedans qui les rend ainsi disciplinables à apprendre» (II. ch. 12.441d). Cette voix animale, en harmonie avec le monde n'est plus frappée de suspicion. Au contraire elle est reliée à cette musique inhumaine et apaisante «that crept by me upon the waters» (The Tempest, I.2.392) que Ferdinand avait été le premier à entendre et qui pourrait bien faire écho au souffle de Dieu qui dans les premiers versets de la Genèse courait sur la mer avant que Dieu ne prononce ses premiers mots. Les bruits de l'île ont leur source dans la terrible cacophonie de toutes sortes d'oiseaux «hovering in the air and over the sea (making) a strange hollow and harsh howling» dont il est question dans la lettre de Strachey sur le naufrage des Bermudes ${ }^{42}$. Les voix de l'île sont décrites de la même manière: «a strange hollow and 
confused noise» (The Tempest, IV.1.138-9). On voit qu'il y a télescopage possible entre le texte de Strachey et cette confusion «confused sound» dans The Faerie Queene qui décrivait la voix de l'homme sauvage. Et voilà que dans Strachey les marins se mettent à imiter ce terrible bruit d'oiseaux pour piéger justement les oiseaux faisant fi de la mise en garde de Platon. Mais ne fait-on pas chanter désormais les animaux dans les Masques ? Pensons par exemple à la chanson de la mouche dans le Masque d'Obéron. Un dialogue entre l'homme et l'oiseau s'instaure. De cette imitation animale nait la voix polyphonique de The Tempest. C'est la voix d'Ariel, cet être des espaces intermédiaires, ce démon du néo-platonisme à qui Prospero ordonne d'imiter les voix animales et les «voix» des éléments. Son chant sans parole est la voix de l'oiseau, de la harpie, de l'ivrogne, de la nymphe de la mer. La voix devient mer, vent, tonnerre pour dire le nom de Prospero :

\section{Alonso. Methought the billows spoke and told me of it}

The winds did sing it to me ; and the thunder

That deep and dreadful organ pipe pronounced

The name of Prosper : it did bass my trespass.

(The Tempest, III.3.96-9)

Saint Augustin comparait l'univers à un concert ${ }^{43}$ rachetant ainsi par cette métaphore ceux qui se permettent de telles transgressions. Ici la voix de basse du tonnerre est la voix de la culpabilité mais aussi de la rédemption qui fonde l'harmonie retrouvée. Iris, la polyphonique (les sept couleurs de l'arc-en-ciel sont comme des voix de l'univers correspondant aux sept voyelles de l'alphabet grec) présente le Masque. Sa voix reliant le ciel et la terre ${ }^{44}$ est comme celle d'Antoine "propertied / As all the tuned spheres» (Antony and Cleopatra, V.2.83).

The Tempest illustre ce que Gilles Deleuze appelle la déterritorialisation des voix d'une espèce à une autre qui est la naissance de l'harmonie: «La question de la musique est celle d'une puissance de déterritorialisation qui traverse la Nature, les animaux, les éléments et les déserts non moins que l'homme» ${ }^{45}$. Au verbe imiter, il faut substituer le verbe devenir. Ainsi la logique ovidienne prend le pas sur la logique platonicienne. Les métamorphoses d'Ariel dépassent la transgression de l'imitation car ces métamorphoses sont ici christianisées tout comme la pensée platonicienne l'avait été. The Tempest se termine comme L'Apologie de Raimond Sebond se terminait, par une description du 
pouvoir de la grâce définie comme une «divine et miraculeuse métamorphose». La voix humaine si elle peut se creuser ou hurler, pour avoir inspiré à saint Augustin sa théorie du temps devient ici l'artisan de cette métamorphose. Au même moment, elle se met à chanter sur la scène baroque.

Toute l'cuvre de «mellifluous and honey-tongued» (F. Meres, Palladis Tamia, 1598) Shakespeare, orchestre les modulations de voix multiples, voix suspectes, souterraines, voix de la folie et de l'animalité qui dénouent le lien sacré entre le geste et la parole, voix qui ne savent pas parler et qui résonnent en échos infinis; mais voix justes aussi, sceaux de l'intégrité et de la spécificité humaine, voix qui fondent l'ordre de la cité pour être marquées de l'empreinte même de la loi du temps, voix sacrées qui rivalisent avec le silence du crocodile. Dans The Tempest ces voix discordantes finissent par s'harmoniser dans l'univers-concert de l'île de Prospero.

Margaret JONES -DAVIES Université de Paris IV

\section{NOT E S}

${ }^{1}$ A. Artaud, Lettre à Henri Parisot, Lettres de Rodez, in G. Deleuze, Logique du Sens, Paris, Minuit, 1969, p. 103.

${ }^{2}$ G. Deleuze, Mille Plateaux, Paris, Minuit, 1980, p. 309. Le livre d'E. de Fontenay, Le silence des bêtes. La philosophie à l'épreuve de l'animalité, Fayard, 1998, contient une analyse de la pensée de Deleuze sur ce sujet (p. 33-7).

${ }^{3}$ Plutarque, Isis et Osiris, 75B.

${ }^{4}$ L. Ménard, Hermes Trismégiste, Éd. de la Maisnie, 1977, p. 16.

${ }^{5}$ L. Ménard, op. cit., p. 4.

${ }^{6}$ P. Zumthor, La Lettre et la Voix, Paris, Seuil, 1987, p. 276.

${ }^{7}$ P. Zumthor, op. cit., p. 276.

${ }^{8}$ Montaigne, Euvres complètes, ed. M. Rat, La Pléiade, Paris, Gallimard, 1962, p. 431c.

${ }^{9}$ Cicéron, De Oratore, I.lix.251; Brutus, 84, 290 (cité par B. Vickers, in 
In Defence of Rhetoric, Clarendon Paperbacks, 1990 [1988], p. 37). Sur la réhabilitation de l'acteur par les Stoïciens, voir V. Goldschmidt, Le Système stoïcien et l'Idée de Temps, Vrin, 1977, p. 181.

${ }^{10}$ Voir la communication de C. Bardelmann, «L'écho ou la métamorphose des sens», dans le cadre du séminaire TIMÉE, ENS rue d'Ulm, 31 mai, 1997.

${ }^{11}$ Selon A. Compagnon, Nous, Michel de Montaigne, Paris, Seuil, 1980, p. 222.

${ }^{12}$ A. de Libera, La Querelle des Universaux, Seuil, 1996, p. 142-6. La première occurrence de l'expression «flatus vocis» recensée dans le Thesaurus linguae latinae est dans l'œuvre de Publius Nigidius Figulus (9845 av. J.C.), néo-pythagoricien latin, ami de Cicéron.

${ }^{13}$ J. Jolivet, Arts du langage et théologie chez Abélard, Vrin, 1982, p. 23.

${ }^{14}$ Cité par A. Compagnon, op. cit., p. 70.

${ }^{15}$ Aristote, Rhétorique (III.1405a): «Les homonymes sont utiles au sophiste. Ce sont eux qui lui permettent des supercheries; les synonymes au poète». Voir A. Compagnon, op. cit., p. 53 \& 60.

${ }^{16}$ L. Ménard, op. cit., p. 146. F. Yates, Giordano Bruno and the Hermetic Tradition, Routledge \& Kegan Paul, 1964, p. 9.

${ }^{17}$ Cicéron, De Oratore (III.216).

${ }^{18}$ F. Kermode, The Sense of an Ending, O.U.P., 1967, p. 84-9.

${ }^{19}$ A. Michel, «La parole et la beauté dans la conception augustinienne de l'enseignement», in Saint Augustin, Les Dossiers H, L'Âge d'Homme, 1988 , p. 111.

${ }^{20}$ J. Delumeau, «Faillite de la rédemption», in Saint Augustin, p. 65.

${ }^{21}$ A. Hamilton, «Humanists and the Bible», in J. Kraye, ed., Renaissance Humanism, C.U.P., p. 100.

${ }^{2}$ A. Michel, op. cit., p. 111. G. Venet, Temps et vision tragique, Shakespeare et ses contemporains, Université de la Sorbonne Nouvelle, Paris III, 1985 (sur l'influence de saint Augustin dans l'œuvre de Shakespeare).

${ }^{23}$ A. Michel, op. cit., p. 110.

${ }^{24}$ B.P. Copenhaver \& C. B. Schmitt, Renaissance Philosophy, O.U.P., 1992, p. 179.

${ }^{25}$ B.P. Copenhaver \& C. B. Schmitt, op. cit., p. 96.

${ }^{26}$ B.P. Copenhaver \& C. B. Schmitt, op. cit., p. 96.

${ }^{27}$ G. de Lagarde, La Naissance de l'esprit laique au déclin du moyen âge (volume 3), Droz, 1942, p. 308.

${ }^{28}$ G. de Lagarde (volume 6), op. cit., p. 138.

${ }^{29}$ G. de Lagarde (volume 3), op. cit., p. 381.

${ }^{30}$ Érasme, cité par F. Kiefer, Writing on the Renaissance Stage, Delaware, 1996, p. 13 (note p. 297 : in The collected Works of Erasmus, University of Toronto Press, 1974, 31, p. 161). 
31 M. Jones-Davies, «De l'Illyrie à l'Égypte, vers l'Épiphanie de Twelfth Night», in H. Suhamy (éd.), Twelfth Night, Paris, Ellipses, 1995, p. 78-92.

32 A. Manguel, A History of Reading [1996], Flamingo, 1997, p. 51.

33 B. Vickers, In Defence of Rhetoric, Clarendon Paperbacks, O.U.P., 1988, p. 274.

${ }^{34}$ P. Brockbank, ed., Coriolanus, The Arden Shakespeare, Methuen, 1976, p. 161 , note 174 .

35 G. de Lagarde (volume 3), p. $27 \& 64$.

${ }^{36}$ P. Brockbank (op. cit., note 34), p. 45.

37 A. Alciat, Toutes les Emblèmes (Édit. 1558 et 1564), Aux Amateurs de Livres, Paris, 1989, p. 229-230. Voir M.-M. Martinet. «Sermons in Stones», communication faite au colloque S.I.R.I.R., novembre 1998. A paraître.

38 A. Alciat, op. cit., p. 231.

39 Dante, De vulgari Eloquentia, in Dante, Euvres Complètes, A. Pezard (éd.), La Pléiade, Gallimard, 1965, p. 556.

${ }^{40}$ E. Konigson, «Le Masque du Démon. Phantasmes et métamorphoses sur la scène médiévale», in Le Masque, du Rite au Théâtre, Paris, Éd. du C.N.R.S., 1985, p. 108-10.

${ }^{41}$ S. Greenblatt, Learning to curse [1990], New York, London, Routledge, 1992, p. 20 \& 21-3. Voir aussi l'ouvrage de Terence Hawkes, Shakespeare's Talking Animals, London, 1973, auquel Greenblatt se réfère.

${ }^{42}$ The Strachey Letter, in The Tempest, S. Orgel, ed., The Oxford Shakespeare, Oxford World's Classics, 1998 (1987), p. 215.

${ }^{43}$ Saint Augustin, De civ. Dei, XIX.13, cité par G. de Lagarde (volume 3), p. 32-3.

4 Y. Peyré, «Les Masques d'Ariel, Essai d'interprétation de leur symbolisme», Cahiers Élisabéthains, n²19, 1981, p. 54.

${ }^{45}$ G. Deleuze, op. cit. , p. 380.

Références bibliographiques

Platon, Timée, in Platon, Euvres complètes, tome V, tr. E. Chambry, Paris, Garnier, 1950. 1993.

Platon, La république, tr. P. Pachet, coll. Folio/Essais, Paris, Gallimard,

Aristote, Rhétorique, tr. M. Dufour, Paris, Les Belles Lettres, 1967.

Aristote, De l'âme, tr. J. Tricot, Paris, Vrin, 1995.

Cicéron, De l'orateur, tr. E. Courbaud, Paris, Les Belles Lettres, 1985.

Shakespeare, The Arden Shakespeare, Methuen. 